\title{
Stable Temperature Characteristics and Suppression of Efficiency Droop in InGaN Green Light-Emitting Diodes Using Pre-TMIn Flow Treatment
}

\author{
Ya-Ju Lee, Yi-Ching Chen, Chia-Jung Lee, Chun-Mao Cheng, Shih-Wei Chen, and Tien-Chang Lu
}

\begin{abstract}
We present experimental results on the improved performance and high stable temperature characteristics of the InGaN green light-emitting diode (LED) with pre-trimethlyindium (pre-TMIn) flow treatment. By using pre-TMIn flow treatment, a relatively large radiative coefficient $\left(B=3.34 \times 10^{-11} \mathrm{~cm}^{3} \cdot \mathrm{s}^{-1}\right)$ corresponding to a $9.2 \%$ enhancement in the internal quantum efficiency, as well as a significant reduction of leakage paths for injected carriers, was obtained. Most important, the pre-TMIn flow treatment evidently reduces the dependence of the external quantum efficiency on temperature and efficiency droop of green LEDs. The improvement is thought to be attributable to the preferential formation of In-rich dots upon pre-TMIn flow treatment, which effectively suppresses the trapping of excitons by threading dislocations and the overflowing of injected carriers outside the active regions at elevated temperatures.
\end{abstract}

Index Terms-Efficiency-droop, light-emitting diode.

\section{INTRODUCTION}

$\mathbf{T}$ HE development of InGaN alloys is of priority concern to production of light-emitting diodes (LEDs) that are highly efficient across the visible spectrum and so can deliver the color quality of traditional light sources. In particular, InGaN material systems can be applied to manufacture a high-power blue LED with an external quantum efficiency (EQE) of as high as 56\% [1]. However, the peak EQE of green LEDs is significantly lower than that of InGaN-based blue and AlGaInP-based red LEDs, which characteristic is called the "green gap." Additionally, the efficiency of green LEDs falls more rapidly than blue LEDs as the injected current increases [2]. Therefore, various approaches were developed over the last decade to overcome the aforementioned issues for InGaN-based green LEDs. To engineer a narrow bandgap that is suited to light emission in the green region of the spectrum $(\lambda=500-570 \mathrm{~nm})$, high indium contents of up to $30 \%$ in the quantum wells (QWs) of LEDs are necessary. Such high indium contents are associated with a complex array of properties that evolve with the indium contents of QWs and adversely

Manuscript received April 22, 2010; revised May 21, 2010; accepted June 12, 2010. Date of publication June 21, 2010; date of current version August 06, 2010. This work was supported by the National Science Council of Republic of China (R.O.C.) in Taiwan under Contract NSC-98-2112-M003-001-MY2 and Contract NSC 96-2221-E-009-094-MY3.

Y.-J. Lee, Y.-C. Chen, C.-J. Lee, and C.-M. Cheng are with the Institute of Electro-Optical Science and Technology, National Taiwan Normal University, Taipei 116, Taiwan (e-mail: yajulee@ntnu.edu.tw; easywindchen@gmail.com; maikuraki25@gmail.com; diablo2924@pchome.com.tw).

S.-W. Chen and T.-C. Lu are with the Department of Photonics, National Chiao Tung University, Hsinchu 300, Taiwan (e-mail: macub86@yahoo. com.tw; timtclu@mail.nctu.edu.tw).

Color versions of one or more of the figures in this letter are available online at http://ieeexplore.ieee.org.

Digital Object Identifier 10.1109/LPT.2010.2053530 affect crystalline quality and the output performance of LEDs. In addition, for practical application of LEDs, stable temperature dependence on device performance is required. Recently, a few studies have considered improving the interface abruptness and optical properties of green LEDs by supplying flowing trimethlyindium (TMIn) prior to the growth of InGaNQWs [3], [4]. However, in previous studies, the effects of such pre-TMIn flow treatment on the performance of LEDs have barely been discussed. In this work, we present experimental results on the improved performance and high stable temperature characteristics of the InGaN green LED with pre-TMIn flow treatment. For the presented scheme, several essential properties of an LED such as the internal quantum efficiency (IQE), the leakage current, the efficiency droop, and the temperature characteristics are significantly improved. The potential physical cause for the improved performance was also discussed.

\section{DEVICE FABRICATION}

Green LED structures were grown on $c$-plane sapphire substrates by low-pressure metal-organic chemical vapor deposition. Following the growth of a $\mathrm{GaN}$ nucleation layer at $520{ }^{\circ} \mathrm{C}$, a $2-\mu \mathrm{m}$-thick undoped $\mathrm{GaN}$ layer and a $2.5-\mu \mathrm{m}$-thick Si-doped n-type GaN cladding layer were grown at $1050{ }^{\circ} \mathrm{C}$. The active region comprises six periods of a nominally undoped 3-nm-thick $\operatorname{In}_{0.30} \mathrm{Ga}_{0.70} \mathrm{~N} / 14$-nm-thick $\mathrm{GaN}$ multiple quantum wells (MQWs) structure. The growth temperature for $\mathrm{InGaN}$ well and GaN barrier were $750{ }^{\circ} \mathrm{C}$ and $900{ }^{\circ} \mathrm{C}$, respectively. During temperature ramping, a 2-nm-thick GaN capping layer was grown on the well layer to prevent the decomposition of InGaN well layer. An interruption between the well and barrier layers was used to stabilize the reactor temperature and gas flows. During the interruption of growth, a TMIn flow of $60 \mathrm{sccm}$ was introduced into the reactor prior to the growth of an $\mathrm{InGaN}$ well for 10-s duration. Beneath the MQWs structure, there are strain-relaxation layers with seven periods of 3.5-nm-thick $\mathrm{In}_{0.1} \mathrm{GaN}_{0.9} \mathrm{~N} / 3.5$-nm-thick $\mathrm{GaN}$ superlattices. For comparison, a conventional sample obtained by not introducing TMIn flow prior to the well layers was grown. Finally, a 300-nm-thick p-type GaN cladding layer was grown on top of the MQWs. The grown wafers were then fabricated to LED devices with chip size of $300 \times 300 \mu \mathrm{m}^{2}$ for subsequent measurements.

\section{RESUlT AND DisCUSSION}

Fig. 1 presents cross-sectional transmission electron-microscopic (TEM) images of the MQWs that were prepared (a) without and (b) with pre-TMIn flow treatment, respectively. The boundaries of the InGaN-GaN QWs in both samples are 


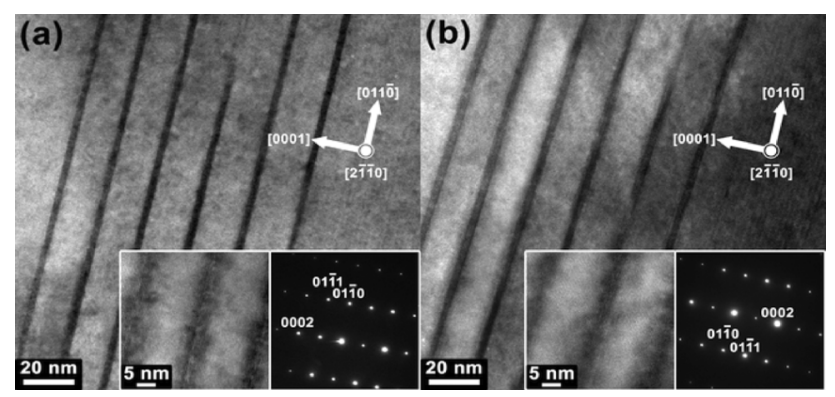

Fig. 1. Bright-field TEM images of InGaN-GaN MQWs grown (a) without and (b) with pre-TMIn flow treatment. Inset: TEM diffraction patterns and enlarged TEM images of InGaN well regions.

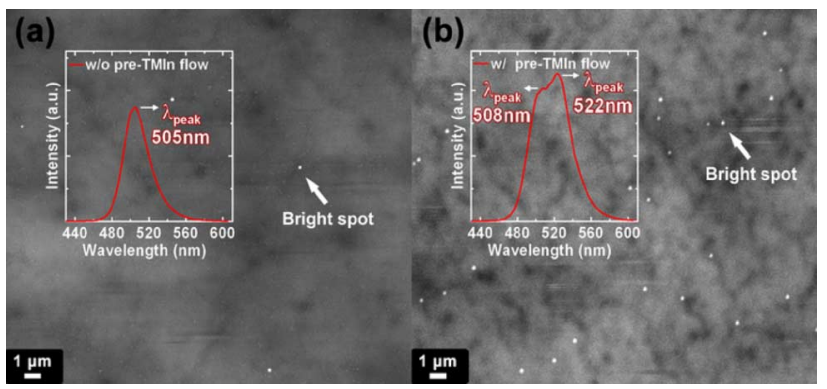

Fig. 2. CL images of MQWs prepared (a) without and (b) with pre-TMIn flow treatment. Inset: CL spectra with peak wavelengths.

clear and well defined, suggesting that the pre-TMIn flow treatment slightly affects interface abruptness. TEM diffraction patterns are inset in the figure to elucidate the crystallographic orientations of both samples. Also inset are high-magnification TEM images, which reveal segregations of indium atoms along the (0001) growth direction in both samples, suggesting the desorption of indium and its subsequent penetration into the GaN barrier layer remains in the sample that underwent pre-TMIn flow treatment.

To analyze the spatial characterization of MQWs, cathodoluminescence (CL) was measured using a Jeol-7000F SEM that was equipped with a Gatan Mono-CL system. Fig. 2 displays CL images of MQWs that were prepared (a) without and (b) with pre-TMIn flow treatment, respectively. The density of bright spots (indicated by an arrow) randomly distributed over the sample that had undergone pre-TMIn flow treatment, apparently exceeded that of those over the conventional sample, and the area of bright emission was much larger. These effects are possibly attributable to the fact that supplying TMIn flow when the growth was interrupted caused randomly distributed $\mathrm{InN}$ clusters to form on the surface of the GaN barrier layer. These InN clusters functioned as seeds for the subsequent indium of InGaN well layer, promoting the incorporation of indium atoms in MQWs and the formation of localized In-rich dots or regions; radiative efficiency was also improved. The CL spectra (inset in Fig. 2) are consistent with the above analysis, a stronger emission intensity with an additional peak are obtained at a long wavelength $(\lambda=522 \mathrm{~nm})$ from the sample with pre-TMIn flow treatment. To compare quantitatively the radiative efficiency of the samples, room-temperature power-dependent photoluminescence (PL) measurements, first made by Dai et al., were made herein to determine their IQE as a function of carrier concentration [5], as shown in Fig. 3(a). The inset presents PL
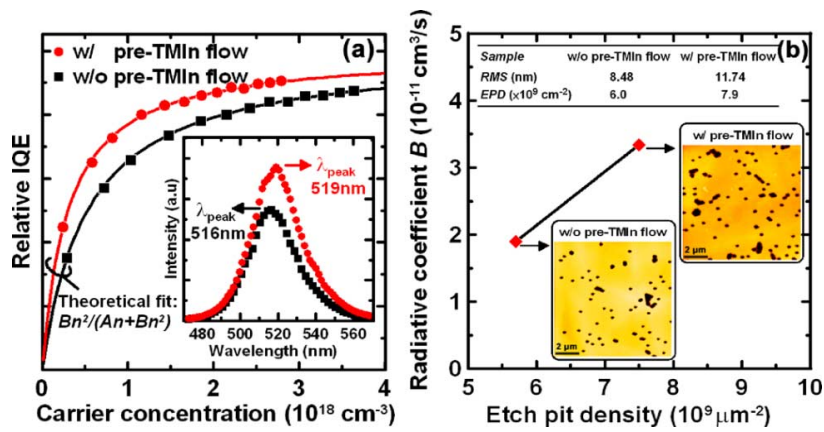

Fig. 3. (a) IQE versus carrier concentration. Inset: PL spectra with peak wavelengths. (b) Radiative coefficient $B$ versus etch pit density. Inset: AFM images of surface morphology following etching treatment with phosphoric acid.

spectra of both samples, which are similar to CL spectra. Apparently, the IQE of the sample that had undergone pre-TMIn flow treatment considerably exceeds that of the conventional sample throughout the carrier concentration region. Pre-TMIn flow treatment increased the IQE by approximately $9.2 \%$ at a typical carrier concentration of $2 \times 10^{18} \mathrm{~cm}^{-3}$. The relationship between measured IQE and carrier concentration for both samples is well described by IQE $=B n^{2} /\left(A n+B n^{2}\right)$, where $A$ and $B$ are the nonradiative and radiative coefficients, respectively. If a reasonable value of $A=3 \times 10^{7} \mathrm{~s}^{-1}$ is assumed [6], then $B$ can be obtained. The calculated values of the samples without and with pre-TMIn flow treatment are $B=1.9 \times 10^{-11} \mathrm{~cm}^{3} \cdot \mathrm{s}^{-1}$ and $B=3.34 \times 10^{-11} \mathrm{~cm}^{3} \cdot \mathrm{s}^{-1}$, respectively. Fig. 3(b) plots the radiative coefficient $B$ against etch pit density (EPD). Etch pits were formed by etching with of phosphoric acid, and the EPD values of the samples without and with pre-TMIn flow treatment that were estimated from the AFM images in the inset in Fig. 3(b) were $5.7 \times 10^{9} \mathrm{~cm}^{2}$ and $7.5 \times 10^{9} \mathrm{~cm}^{2}$, respectively. High EPD generally corresponds to a high density of threading dislocations, and therefore, a small value of $B$. However, the samples herein exhibited the opposite tendency. Watanabe et al., however, suggested that the pits may be originated by local lattice expansion because of the presence of In-rich dots in MQWs, rather than being connected to the threading dislocations [7]. Accordingly, as previously discussed in reference to Fig. 2(b), a high density of In-rich dots distributed over MQWs is expected in the sample that underwent pre-TMIn flow treatment. The strong spatial localization of In-rich dots effectively prevents the capture of excitons by threading dislocations, increasing $B$ and the IQE.

Temperature dependence on the output performance of the samples is now considered. Fig. 4 plots the $I-V$ characteristics of the samples (a) without and (b) with pre-TMIn flow treatment on a semilog scale from $T=30^{\circ} \mathrm{C}$ to $T=150{ }^{\circ} \mathrm{C}$ at intervals of $30^{\circ} \mathrm{C}$. The $I-V$ curves of both samples exhibit the typical current rectification of InGaN-based LEDs. The series $\left(R_{S}\right)$ and parallel $\left(R_{P}\right)$ resistances, and the ideality factor $\left(n_{\text {ideal }}\right)$ of the samples at all temperature were extracted and presented in Fig. 4 as well. At a given temperature, the values of $R_{S}$ and $n_{\text {ideal }}$ for both samples are similar, suggesting that the pre-TMIn flow treatment barely affected the transport property of injected carriers under forward bias injection. Furthermore, both $R_{S}$ and $n_{\text {ideal }}$ slowly declined as the temperature increased, mainly because of the improvement of the p-type transport properties as 


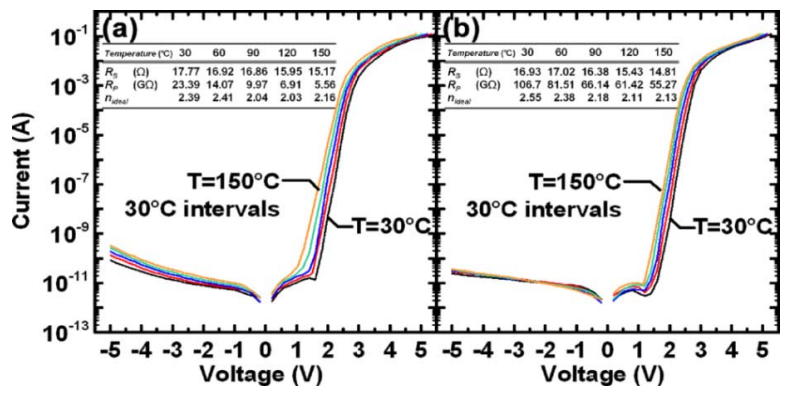

Fig. 4. $\ln |I|$ versus $V$ for the sample (a) without and (b) with pre-TMIn flow treatment from $T=30{ }^{\circ} \mathrm{C}$ to $T=150{ }^{\circ} \mathrm{C}$. Series $\left(R_{S}\right)$ and parallel $\left(R_{P}\right)$ resistances, and ideality factor $\left(n_{\text {ideal }}\right)$ are also shown.

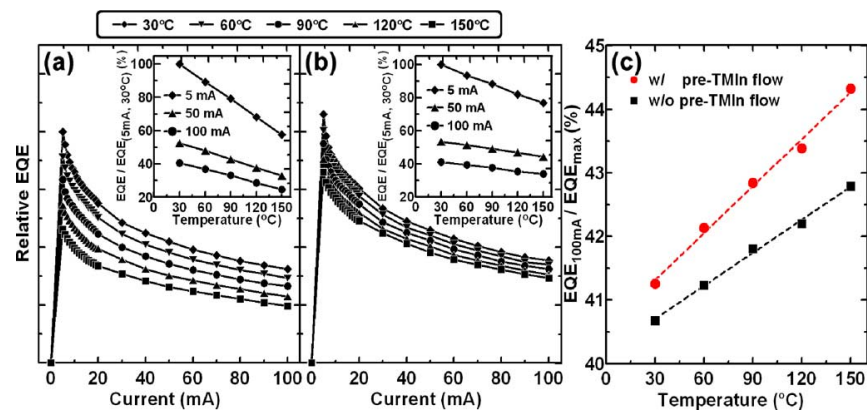

Fig. 5. EQE versus injected current for sample (a) without and (b) with preTMIn flow treatment from $T=30^{\circ} \mathrm{C}$ to $T=150{ }^{\circ} \mathrm{C}$. Inset: Normalized EQE versus temperature at three different injected currents, $I=5 \mathrm{~mA}, I=50 \mathrm{~mA}$, and $I=100 \mathrm{~mA}$. (c) EQE droop versus temperature for both samples.

temperature increased, which promotes the activation of acceptors at elevated temperatures. However, the value of $R_{P}$ of the sample that had undergone pre-TMIn flow treatment considerably exceeds that of the conventional sample, at all temperatures. For example, at $T=30{ }^{\circ} \mathrm{C}$, the extracted $R_{P}$ values are around $R_{P}=23.39 G \Omega$ and $R_{P}=106.70 G \Omega$ for the samples without and with pre-TMIn treatment, respectively. An indium atom can preferentially bond at the core of a threading dislocation, pinning it, preventing it from gliding during growth [8]. Therefore, when pre-TMIn flow is supplied, the number of leakage paths that are associated with the threading dislocations are significantly reduced, resulting in high $R_{P}$ values.

Fig. 5 plots the EQE as a function of injected current for the samples (a) without and (b) with the pre-TMIn flow treatment from $T=30{ }^{\circ} \mathrm{C}$ to $T=150{ }^{\circ} \mathrm{C}$. At all temperatures, the $\mathrm{EQE}$ values of the sample that had undergone the pre-TMIn flow treatment are better than that of the conventional sample. This enhancement increases with temperature. On average, the EQE value is enhanced by $7.5 \%$ at $T=30^{\circ} \mathrm{C}$, increasing to $44.5 \%$ at $T=150{ }^{\circ} \mathrm{C}$. The insets in Fig. 5(a) and (b) plot the normalized EQE versus temperature for both samples. Clearly, the EQE values of the sample that had undergone pre-TMIn flow treatment declined slightly as temperatures increased, demonstrating that the characteristics of this sample were more thermally stable. For example, at an injected current of $I=100 \mathrm{~mA}$, the normalized EQE of the sample that had undergone pre-TMIn flow treatment declined by under $7.2 \%$ as the temperature increased from $T=30{ }^{\circ} \mathrm{C}$ to $T=150{ }^{\circ} \mathrm{C}$; however, that of the conventional sample decreased by $16.0 \%$ under identical conditions. Studies have suggested that the localized injected car- riers in In-rich dots function excellently as radiative recombination centers, effectively suppressing the overflow of injected carriers outside the MQWs at elevated temperatures. This fact indicates that pre-TMIn treatment can substantially reduce the dependence of the EQE on temperature. Finally, Fig. 5(c) plots the EQE droop (EQE at $I=100 \mathrm{~mA}$ divided by the maximum EQE) against temperature for both samples. Less efficiency droop was observed for the sample that had undergone pre-TMIn flow treatment at all temperatures, which is fundamentally due to its much improved confinement of injected carriers in MQWs.

\section{SUMMARY}

In summary, pre-TMIn flow treatment was incorporated in a InGaN green LED to minimize the temperature dependence on the EQE and alleviate the efficiency droop. For temperature in the range of $30^{\circ} \mathrm{C}$ to $150{ }^{\circ} \mathrm{C}$, the $\mathrm{EQE}$ (at $I=100 \mathrm{~mA}$ ) of the sample with pre-TMIn flow treatment drops by less than $7.2 \%$, representing a significant improvement of temperature characteristics as compared to a reduction of $16.0 \%$ in the conventional LED under identical condition. On average, with pre-TMIn flow treatment, the EQE value of an LED is enhanced by $7.5 \%$ at $T=30{ }^{\circ} \mathrm{C}$, increasing to $44.5 \%$ at $T=150{ }^{\circ} \mathrm{C}$. Moreover, the presented device exhibits less efficiency droop at all measured temperatures. We, therefore, conclude pre-TMIn flow treatment is determined to be a promising scheme for the improvement of output performance of InGaN-based green LEDs and bridging the "green gap" in solid-state lighting.

\section{ACKNOWLEDGMENT}

The authors gratefully acknowledge L.-R. Chen for his technical support.

\section{REFERENCES}

[1] M. R. Krames, O. B. Shchekin, M. M. Regina, G. O. Mueller, L. Zhou, G. Harbers, and M. G. Craford, "Status and future of high-power lightemitting diodes for solid-state lighting," IEEE Journal of Display Technology, vol. 3, no. 2, pp. 160-175, 2007

[2] T. Mukai, M. Yamada, and S. Nakamura, "Characteristics of InGaNbased UV/blue/green/amber/red light-emitting diodes," Jpn. J. Appl. Phys., vol. 38, pp. 3976-3981, 1999.

[3] S.-J. Leem, M. H. Kim, J. Shin, Y. Choi, and J. Jeong, "The effects of in flow during growth interruption on the optical properties of InGaN multiple quantum wells grown by low pressure metalorganic chemical vapor deposition," Jpn. J. Appl. Phys., vol. 40, pp. L371-L373, 2001.

[4] M. S. Kumar, J. Y. Park, Y. S. Lee, S. J. Chung, C.-H. Hong, and E.-K. Suh, "Improved internal quantum efficiency of green emitting InGaN/GaN multiple quantum wells by in preflow for InGaN well growth," Jpn. J. Appl. Phys., vol. 47, pp. 839-842, 2008.

[5] Q. Dai, M. F. Schubert, M. H. Kim, J. K. Kim, E. F. Schubert, D. D. Koleske, M. H. Crawford, S. R. Lee, A. J. Fischer, G. Thaler, and M. A. Banas, "Internal quantum efficiency and nonradiative recombination coefficient of GaInN/GaN multiple quantum wells with different dislocation densities," Appl. Phys. Lett., vol. 94, no. Art. 111109, 2009.

[6] Y. C. Shen, G. O. Mueller, S. Watanabe, N. F. Gardner, A. Munkholm, and M. R. Krames, "Auger recombination in InGaN measured by photoluminescence," Appl. Phys. Lett., vol. 91, no. Art. 141101, 2007.

[7] K. Watanabe, J. R. Yang, S. Y. Huang, K. Inoke, J. T. Hsu, R. C. Tu, T. Yamazaki, N. Nakanishi, and M. Shiojiri, "Formation and structure of inverted hexagonal pyramid defects in multiple quantum wells InGaN/ GaN," Appl. Phys. Lett., vol. 82, no. Art. 718, 2003.

[8] S. Yamaguchi, M. Kariya, S. Nitta, H. Amano, and I. Akasaki, "The effect of isoelectronic in-doping on the structural and optical properties of (Al)GaN grown by metalorganic vapor phase epitaxy," Jpn. J. Appl. Phys., vol. 39, pp. 2385-2388, 2000. 